\title{
Quantum Orders from Strong Disorder
}

\section{Featured paper:}

1. Localization protected quantum order, David A. Huse, Rahul Nandkishore, Vadim Oganesyan, Arijeet Pal, S.L.Sondhi. arXiv:1304.1158.

\section{Recommended with a commentary by Ashvin Vishwanath, UC Berkeley}

The ability to isolate quantum many body systems and study their dynamics, such as in ultra cold atomic gases, has raised fundamental questions on the approach to thermodynamic equilibrium in a closed system. Typically, a system with sufficiently large number of degrees of freedom is expected to come to thermal equilibrium (thermalize) even when it is isolated. For quantum systems that thermalize, the Eigenstate Thermalization Hypothesis (ETH) [1] states that thermalization is evident even in a single eigenstate. Expectation values of local physical observable, when taken in an eigenstate, are equal to that in the Gibbs ensemble with the same average energy.

However, not all interacting many bodes systems thermalize. Besides the obvious exception of integrable systems with specially tuned Hamiltonians, a more generic route to realizing a non-thermalizing system is via localization. Historically, Anderson motivated the study of localization in his 1958 paper, in part, by pointing out that it provided a physical example of a non-ergodic system. More recently, localization in interacting many body systems with disorder (or many body localization - MBL) was argued for by Basko, Aleiner and Altshuler. For example, consider a tight binding model of electron hopping in 1D with random site potentials. In the absence of electron-electron interactions, it is well known that all single particle states are localized and a many body eigenstate is simply obtained by occupying a set of localized states. With weak interactions, the localized nature of the eigenstates is argued to remain - i.e. . the many body eigenstates can be thought to be only slightly modified from those for the non-interacting problem.

The absence of thermalization of MBL systems liberates their eigenstates from the ETH, opening the door to new possibilities that go beyond the standard lore of statistical physics. The featured paper explores new phenomena that only appear in the presence of many body localization. 


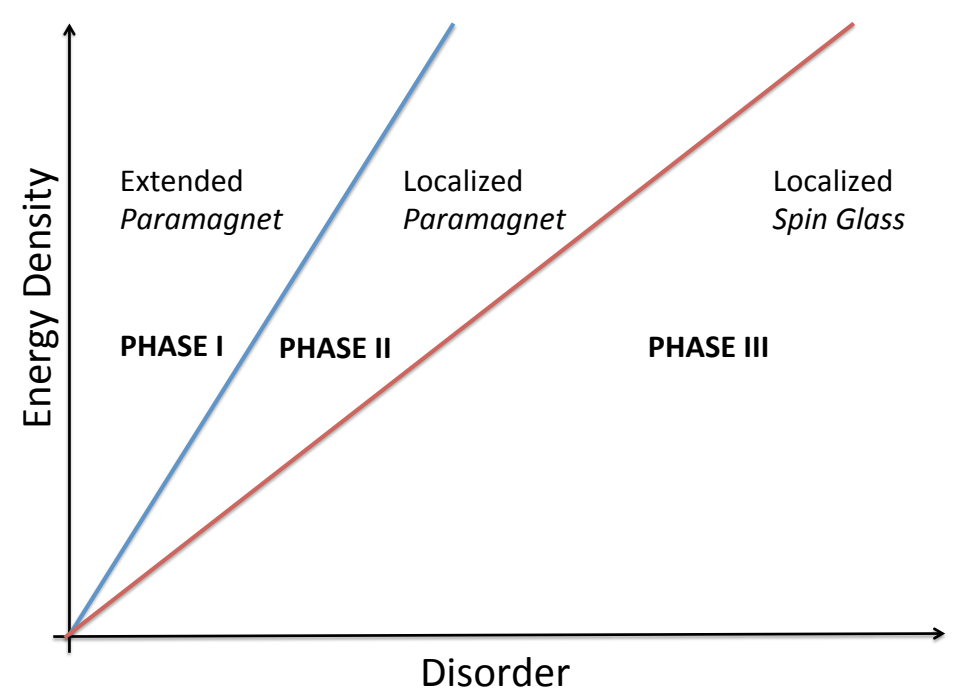

FIG. 1: A schematic phase diagram of the 1D quantum Ising model, adapted from the featured reference. In the absence of disorder, on increasing temperature (vertical axis) one encounters Phase I, a paramagnet where states are extended. Increasing disorder leads first to the MBL paramagnet (Phase II) and then to Phase III which is also localized, but in which the magnetic moments order.

For concreteness, consider a one dimensional system, the quantum Ising chain:

$$
H=-\sum_{i}\left(J_{i} \sigma_{i}^{z} \sigma_{i+1}^{z}+h_{i} \sigma_{i}^{x}\right)+\ldots
$$

where the dots refer to additional terms such as $V \sigma_{i}^{x} \sigma_{i+1}^{x}$. In their absence the problem can be mapped to one of noninteracting fermions which displays special features. The added terms convert this into a generic, interacting Hamiltonian. In the clean limit, when all the couplings are independent of position there are two zero temperature phases - a ferromagnet e.g. at $J \gg h$ and a paramagnet at $J \ll h$. At any finite temperature however the model has a single phase, given the low dimensionality. Thus, excited states which differ from the ground state by a finite energy density, (which corresponds to finite temperature by ETH) evolve smoothly on tuning parameters.

However, the featured reference considers the case of random couplings. With sufficiently strong disorder, when MBL takes over, one can obtain qualitatively new phenomena as sketched in the figure. The vertical axes is energy density, which for a system that thermalizes, can essentially be thought of as temperature. The horizontal axis is disorder strength. 
Say we begin at the origin - a clean system at zero temperature in the ferromagnetic phase. Now, increasing temperature leads to a paramagnet, which is delocalized. This is the Phase I, and in a clean system the only phase at finite temperature. However, consider now increasing disorder, that leads to many body localization. On moving in the horizontal direction one first encounters the localized paramagnet (Phase II). Interestingly, there is a third phase as well - a localized phase with magnetic order. The ordering pattern is spin glass like, since although the $J_{i}$ can be taken positive, one is considering a finite energy eigenstate. By magnetic ordering we mean that spin correlation functions $\left\langle\sigma_{i}^{z} \sigma_{i+r}^{z}\right\rangle$ in an eigenstate acquires long range order in the thermodynamic limit. These phases are established in the featured reference by first studying special limits and then arguing for perturbative stability on including more generic interactions. Thus, there is a transition between MBL states with rather different character (Phase II and Phase III) on tuning disorder strength. It has been argued here and in Ref.[2] that the transition (red line) can be continuous and is also an MBL state, similar to the critical ground state of the disordered problem.

Interestingly, this also implies the persistence of a topological distinction between phases in the high energy eigenstates. The 1D transverse field Ising model can be mapped, via a Jordan Wigner transformation, to neutral fermions that hop and experience pairing on the 1D lattice. The two ground state phases are, in terms of fermions, two superconductors with a topological distinction. The ferromagnet corresponds to a topological phase with Majorana zero modes at the edges, while the paramagnet corresponds to the trivial phase. Usually, one expects that such topological phases are protected by a gap and that any finite temperature will cause their edge modes to leak, destroying their defining properties. However, localizing the bulk excitations leads to a topological distinction between finite energy eigenstates.

Several open questions remain, some of which are being actively studied. How does localization set in as one crosses the transition from an extended to an MBL phase? How can one observe coherent quantum phenomena in these 'hot' systems, which, despite the absence of a true temperature, have a very high energy density? Finally, one can ask whether disorder is an essential ingredient - while to localize noninteracting particles one must have quenched disorder, can an interacting system evade this requirement? 
[1] Deutsch, J. M. Phys. Rev. A 43, 20462049 (1991). Srednicki, M. , Phys. Rev. E 50, 888901 (1994). Marcos Rigol, Vanja Dunjko and Maxim Olshanii Nature 452, 854-858 (2008).

[2] R. Vosk and E. Altman, arXiv:1307.3256. 